\title{
The Effect of Speckle Filtering on Scale-Dependent Texture Estimation of a Forested Scene
}

\author{
Michael J. Collins, Member, IEEE, Jonathan Wiebe, and David A. Clausi
}

\begin{abstract}
Spatial fluctuations in microwave backscatter may be an important piece of information in discriminating tree stands. However, the presence of speckle in synthetic aperture radar (SAR) image data is a barrier to the exploitation of image texture. We explored a new methodology that combines a recent adaptive speckle reduction algorithm by Lopes et al. [12] with a generic texture estimation scheme. We investigated the claim that this filter was capable of preserving backscatter texture. To understand if speckle reduction was destroying backscatter texture, we compared the strength of the relationship between forest inventory parameters and image texture as a function of spatial scale for both filtered and unfiltered images. We used Radarsat Fine mode image data: single look resolution is approximately $8.5 \mathrm{~m}$, and pixel spacing is $3 \mathrm{~m}$. Our study area was northern Vancouver Island, B.C., on the west coast of Canada. For the unfiltered data, we found that the ability of image texture to predict the forest parameters decreased as the texture scale increased from 3 to $13 \mathrm{~m}$, suggesting greater information content in the small scale texture. For the filtered data, this relationship was much weaker at small scales and was not a function of distance. Our results suggest that the speckle filter was not retaining small scale texture, which is consistent with the theoretical hypotheses underlying its multiplicative noise model. We also show that there is significant information in small scale SAR image texture that may be used as an adjunct to other spatial information for discriminating tree stands in the temperate rain forest.
\end{abstract}

\section{INTRODUCTION}

$\mathbf{R}$ ECENT research has demonstrated the potential of SAR image texture in helping to discriminate tree stands on a forested landscape [13], [8]. SAR image texture consists of two components: backscatter fluctuations, which contain information about the scene, and speckle fluctuations, which are produced by the imaging process. The information content of the backscatter texture is driven by a number of factors, of which the three most important are

1) radar wavelength, which determines the penetration of the wave into the forest canopy;

2) transmit/receive polarization state, which is sensitive to certain scattering processes;

3) spatial resolution (and sample spacing), which determines the texture scales that are retrievable from the image.

Our research was an exploration of the information content of RADARSAT fine mode image texture as an aid for constructing

Manuscript received March 31, 1999; revised October 4, 1999.

M. J. Collins is with the Department of Geomatics Engineering, University of Calgary, Calgary, Alta., Canada (e-mail: mjcollin@ucalgary.ca).

J. Wiebe is with the NovAtel, Inc., Calgary, Alta., Canada (e-mail: jwiebe@novatel.ca).

D. A. Clausi is with the Department of Systems Design Engineering, University of Waterloo, Waterloo, Ont., Canada (e-mail: dclausi@engmail.uwaterloo.ca).

Publisher Item Identifier S 0196-2892(00)03927-9. and maintaining forest inventory systems. We have reported the related results elsewhere [20]. The polarization state is $\mathrm{HH}$, the wavelength is about $5.5 \mathrm{~cm}$, the spatial resolution is about 8.5 $\mathrm{m}$ in range and azimuth, and the pixel spacing is about $3 \mathrm{~m}$ (i.e., the data is Nyquist sampled with respect to the resolution cell in range and azimuth). The study site was over the temperate rain forests of northern Vancouver Island, B.C., on the west coast of Canada. A $5.5 \mathrm{~cm}$ wavelength will not penetrate the canopy to any appreciable extent, and the backscatter fluctuations will be driven by the dielectric geometry of the canopy surface. The tree stands in this area have a wide variety of ages and heights and we anticipated that the backscatter texture might contain information over a fairly large range of scales, but that the scales on the order of $10 \mathrm{~m}$ (i.e., near the system resolution) would be important.

In exploratory research, one generally does not know the scales over which the backscatter fluctuations will contain scene information. As the scales approach that of the resolution cell, speckle fluctuations will be comparable to the information-bearing backscatter texture. Hence, texture extraction algorithms must deal with speckle. There have been three broad approaches to this problem.

1) Ignore the speckle and apply generic texture extraction algorithms and discriminate scene texture based on the particular texture features of the algorithm.

This is the approach reported most often in the literature, and the most popular texture estimation algorithm has been based on the grey level co-occurrence matrix. Its use was first documented by Ulaby et al. [19], and it has been used in sea ice texture estimation [1], [2] and analysis of agricultural fields [17].

2) Incorporate both the speckle and the backscatter fluctuations in a data model and discriminate scene texture based on model parameters.

The data model of choice is called the product model, which assumes that the speckle process is independent of the scene processes, giving rise to the backscatter fluctuations. This assumption is equivalent to assuming that the backscatter fluctuations are very smooth, i.e., that the scale of the scene texture is much greater than the scale of the resolution cell. While this assumption is not always valid, the approach is very useful when it is, e.g., [18], [10].

3) Incorporate both the speckle and the backscatter fluctuations in an imaging process model and discriminate scene texture based on model parameters.

This approach is the most sophisticated and is relatively infrequent. It has been spearheaded by Chris Oliver of the 
Defense Research Agency (DRA), London, U.K. He and his colleagues have published a wealth of material that has recently been summarized in a book [16]. This approach includes models of correlated backscatter textures that allow a discrimination based on two models of parameters: the order parameter and the correlation length (although uncertainties in estimates of the latter are relatively high).

A fourth strategy that has not been reported in the literature is to combine a speckle reduction filter with a generic texture algorithm. This has not been feasible until recently, since speckle reduction filters have been designed to restore mean backscatter levels rather than backscatter fluctuations. However, a recent paper by Lopes et al. [11] reports an adaptive filter that claims to preserve backscatter texture as well as to restore mean backscatter (see [11, p. 1753]). While the algorithm is based on the product model, which essentially assumes there is no backscatter texture to restore below a few tens of resolution cells (in our case, no texture at scales below 80-100 m), the claim that it can preserve texture is unqualified. Although skeptical, we were intrigued that this filter offered an entirely new strategy for extracting backscatter texture from SAR imagery. In this paper, we report an examination of the scale-dependent information content of SAR image texture of filtered and unfiltered SAR imagery.

We have two hypotheses that drove the research reported here. First, that the backscatter texture containing information about forest stand parameters is scale dependent, with significant information at scales near the system resolution. Second, that the Lopes et al. speckle filter will destroy backscatter texture at scales near that of the resolution cell.

We will not rehearse the details of speckle properties or speckle filtering. These are well known and can be found in many places in the literature. We provide a brief review of the Lopes filter since its implementation is not entirely straightforward.

\section{A. Enhanced Adaptive Filters}

Lopes et al. [12] reviewed current speckle reduction filters by Lee [9], Kuan et al. [6], [7], and Frost et al. [3] and enhanced their performance by making greater use of the coefficient of variation $C_{I}$, defined as $C_{I}=S T D(I) /\langle I\rangle$. The coefficient of variation $(\mathrm{CV})$ has been noted elsewhere to be a strong function of texture [19]. The enhancement is possible because the Lee, Frost, and Kuan filters are also functions of $C_{I}$ but were not using this variable to its full potential. There are three situations, defined by Lopes et al. [12], which a speckle filter may encounter.

1) Homogeneous area. This is a good example of fully developed speckle and multiplicative noise in which speckle can easily be separated from the scene. The filter in this case should restore the scene cross section exactly like a box filter.

2) Heterogeneous area. In this situation, it is important to preserve the heterogeneous image features such as texture and structure while smoothing speckle. The speckle filter is only applied to the subscenes of the heterogeneous area where all three criteria listed below are valid.

a) Fully developed speckle.

b) Multiplicative speckle model.

c) Speckle and scene variability can be separated successfully.

3) Heterogeneous area without valid model assumptions. The speckle filter should not attempt to reconstruct these areas, because at least one of the filter criteria listed above $(\mathrm{a}, \mathrm{b}$, and $\mathrm{c}$ ) has not been met.

For the following, define $C_{F}$ as the coefficient of variation of a perfectly homogeneous area, $C_{I}$ as the the coefficient of variation of the area being examined, and $C_{\max }$ as the coefficient of variation that would be calculated given situation 3 , listed previously. Based on these three definitions, a speckle filter should perform three operations:

1) Spatial averaging if $C_{I} \leq C_{F}$.

2) Selective spatial averaging if $C_{F}<C_{I}<C_{\max }$.

3) Preserve existing features if $C_{I} \geq C_{\max }$.

$C_{F}$ has been theoretically determined for homogeneous areas from intensity L-look images to be $1 / \sqrt{L}$ [19], [12]. If determined from homogeneous portions of an image $C_{F}$ is distributed as a pseudo-Gaussian function [12].

$C_{\max }$ is not easily determined. There is no theoretical threshold for $C_{\max }$. If $C_{\max }$ is chosen too large than valuable textural information may be lost because the filter may choose to smooth the area. It may be best to determine $C_{\max }$ from a textured area to ensure that area is preserved.

Lopes et al. [11] reported a new adaptive speckle reduction filter that was capable of recognizing and preserving backscatter texture as well as image structure such as strong scatterers, lines and edges. This filter uses a Bayesian approach with the multiplicative speckle model and a gamma distributed radar cross section. Two thresholds are used in the algorithm: $C_{\max }$ and $C_{F}$. If the local $\mathrm{CV}$ is greater than $C_{\max }$, the window is textured or contains structure. If the local $\mathrm{CV}$ is less than $C_{F}$, the window is homogeneous.

Since this algorithm explicitly uses the multiplicative model $I=R \times F$, where $I=$ intensity, $R=$ reflectivity, and $F=$ speckle, it is critical to locate heterogeneous areas where this assumption may not be valid. If the area is heterogeneous, it may contain structure or texture. The geometrical ratio detectors are able to detect the heterogeneous areas with structure (edges, lines, and points). The local $\mathrm{CV}$ is able to detect heterogeneous areas with texture. Initially, a large window is used and assumed homogeneous. It may iteratively become smaller if the window contains edges or lines, until the edge or line is avoided by the window. However, speckle is reduced more effectively with a larger window size, so one should limit how small the window can become. Once the window is considered homogeneous or the window cannot get any smaller, the reflectivity of the center image cell is estimated with the adaptive gamma-gamma MAP (GGMAP) filter. If a point target is detected, the image cell intensity is preserved. 
Using the Bayesian approach to restore the image, the so called gamma-gamma MAP solution [one gamma for $p(\sigma)$ and one gamma for $p(I \mid \sigma)$ ] is given by

$$
\begin{aligned}
& R_{\mathrm{MAP}} \\
& =\frac{(\alpha-L-1)\langle R\rangle+\sqrt{\langle R\rangle^{2}(\alpha-L-1)^{2}+4 \alpha L I\langle R\rangle}}{2 \alpha}
\end{aligned}
$$

where $R(=\sigma \eta)$ is the local reflectivity, $R_{\mathrm{MAP}}$ is the MAP estimate of the local reflectivity, $I$ is the intensity, $L$ is the number of looks, $\alpha=\left(1+\left\langle C_{F}\right\rangle^{2}\right) /\left(\left\langle C_{I}\right\rangle^{2}-\left\langle C_{F}\right\rangle^{2}\right), C_{F}=1 / \sqrt{L}$ is the coefficient of variation for pure speckle, and $C_{I}=\sigma_{I} /\langle I\rangle$ is the coefficient of variation for a window. In practice, $\langle R\rangle$ is estimated by $\langle I\rangle$.

If the scene is homogeneous, then $R \rightarrow\langle I\rangle$. Thus, we get $\alpha \rightarrow \infty$ (or $C_{I} \rightarrow C_{F}$ ) and our estimate $R_{\mathrm{MAP}} \rightarrow\langle R\rangle$. This makes sense because a good filter for a homogeneous scene is an averaging filter. Conversely, when $L \rightarrow \infty$, then $R_{\mathrm{MAP}} \rightarrow I$, because a large number of looks effectively reduces the speckle.

\section{DATA AND STUDY SITE}

The Radarsat SAR image obtained for this study is a scene of northern Vancouver Island. The forest stands analyzed here are located along the northern coast of Vancouver Island between Port Hardy and Port McNeill. The land is operated under a Tree Farm License (TFL) to Western Forest Products Ltd. (WFP), Vancouver Island. The Radarsat scene was imaged on December 6, 1996, with beam type F2 (single look) during a rainstorm. The scene is centered at $56^{\circ} 32^{\prime}$ latitude and $127^{\circ} 15^{\prime}$ longitude. The nominal resolution is $6 \mathrm{~m}$ in range and $8.9 \mathrm{~m}$ in azimuth. The image was oversampled twice in range and three times in azimuth for a pixel spacing of $3.125 \mathrm{~m}$.

The reference data for this study were contained in a Geographical Information System (GIS), which contained labeled polygons whose attributes were the forest inventory parameters provided by WFP. The polygons were constructed using the traditional method of air photo interpretation. Hence, our reference data is somewhat unorthodox. Rather than compare our results with ground observations, we are comparing them with the results of current practice. It would be useful of course, to compare our results to ground observations of species, height age, and stand density. However, our intent here is to explore the feasibility of replacing current practice (i.e., air photo interpretation), with an automated SAR image interpretation, which the forest industry can buy from Radarsat International, Richmond, B.C., Canada, for considerably less than the costs of acquiring air photos.

Each polygon represents the boundary of a forest stand that has at least one unique characteristic that distinguishes it from the neighboring polygons. Polygon area, perimeter, year established, species composition, age, height, basal area, stocking, UTM coordinates, and other information are stored as attribute data for each polygon. This study uses only species composition, age, height, and stocking data. Fig. 1 displays the Radarsat image showing the three GIS coverages F64, F63, and F54 used in this research.
The forest stands (outlined by polygons) consist of many different species including Balsam, Western Red Cedar, Yellow Cedar, Alder, Douglas Fir, Hemlock, Lodgepole Pine, and Sitka Spruce. The majority of the forest stands are composed of multiple species. The most frequently occurring species are Western Red Cedar and Western Hemlock. The age of the trees ranged from 0 to $250+$ years. Each stand was categorized into a stocking (trunk density) class: dense, normal, or open. Normal stocking is most common for these stands. Finally, height was also determined and ranged from 1 to $80 \mathrm{~m}$.

The entire Radarsat image covers two biogeoclimatic zones: Coastal Western Hemlock and subalpine Mountain Hemlock [14]. The annual precipitation is more than $350 \mathrm{~cm}$. The old growth forests have structural characteristics: snags, dead tops, conifers with dome-shaped crowns, and spire-shaped crowns of maturing trees [14].

We defined a virtual forest to be a set of texture features from windows for which all but one of the forestry parameters are identical. A virtual forest stand is a set of texture features from windows with identical forestry characteristics. The virtual forest stands make up a virtual forest. For example, suppose all texture features from windows with identical age, species, and height were gathered and assigned to a virtual forest. In this particular virtual forest, every stand is identical in age, species, and height, but not stocking. Stocking is allowed to vary. Now, to test if texture is a function of stocking, one simply has to examine how texture changes as stocking changes within this virtual forest. The virtual forest concept is simply a construct to help organize the forest data into testable subsets.

\section{METHODS}

\section{A. Speckle Filtering}

In this research, we were interested in exploring the information-content of SAR image texture rather than developing an operational algorithm. Hence, we avoided boundary effects by selecting texture windows that lay entirely within stand poygons, so that all pixels involved in a single texture estimate had the same properties. Hence, it was not necessary to implement the full structure detection algorithm described in [11]. However, point target detection was retained to preserve the bright backscatter caused by defoliated tree tops [15]. Fig. 2 outlines our implementation of the GGMAP filter.

The local coefficient of variation is used in the GGMAP speckle filter to determine if more or less smoothing of the image is required. The theoretical value of $C_{F}$ for a one-look intensity image is 1 . The RADARSAT image is an amplitude image, but by squaring the digital number (DN), it was transformed to an intensity image for speckle-filtering purposes. However, the mean of our data's $C_{I}$ is about 0.92 less than $C_{F}$. Since it is impossible for our image to be close to pure speckle, some of the homogeneous targets were examined (because they should be close to pure speckle). The lakes and ocean gave CV values of about 0.9 . Since the weather was stormy on the day the scene was imaged, the water must have been rough, giving the assumed homogeneous targets (water) heterogeneous features. Therefore, there is no absolute way to determine the level of speckle in the image. 


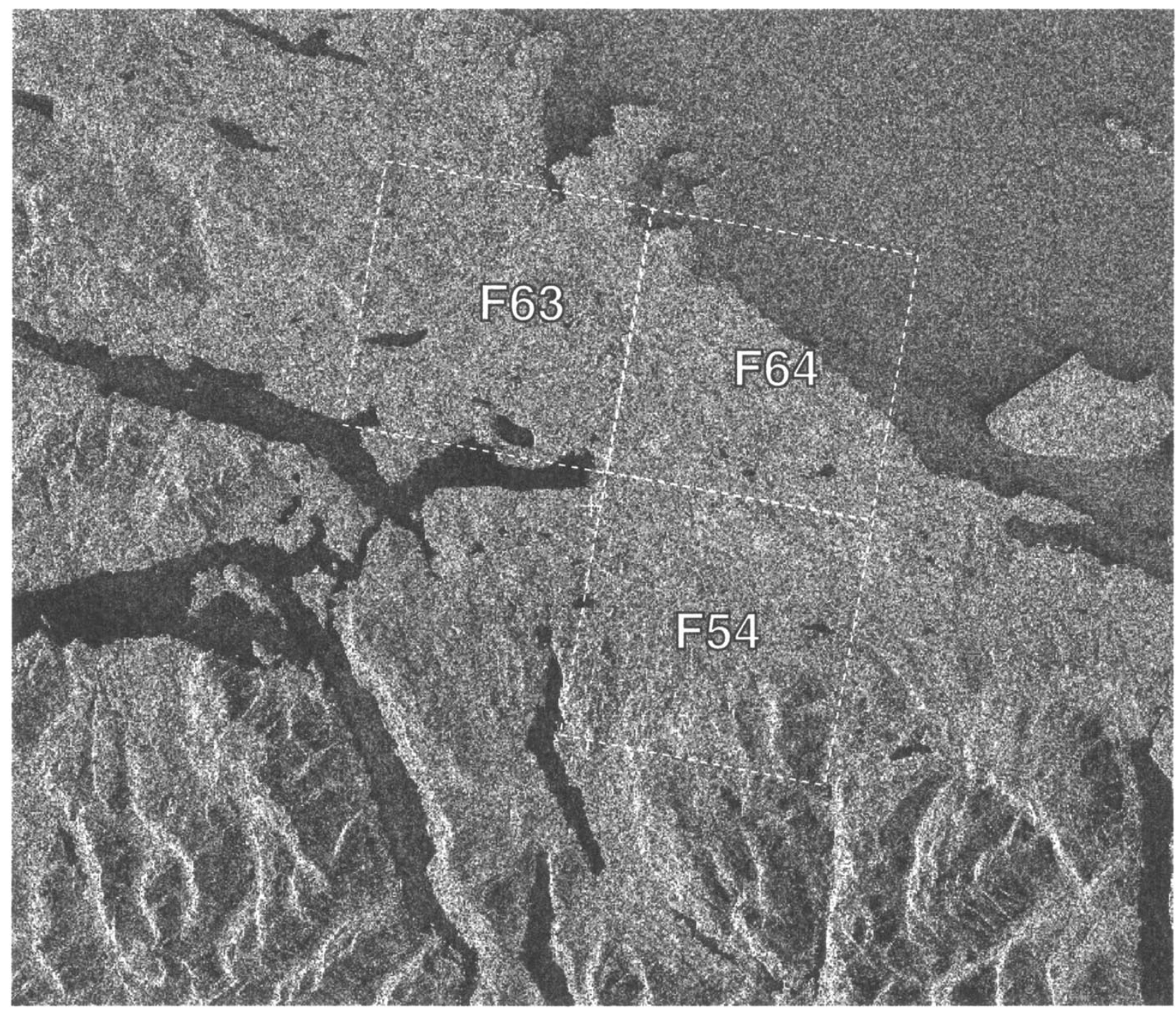

Fig. 1. Raw Radarsat image with three GIS coverages outlined.

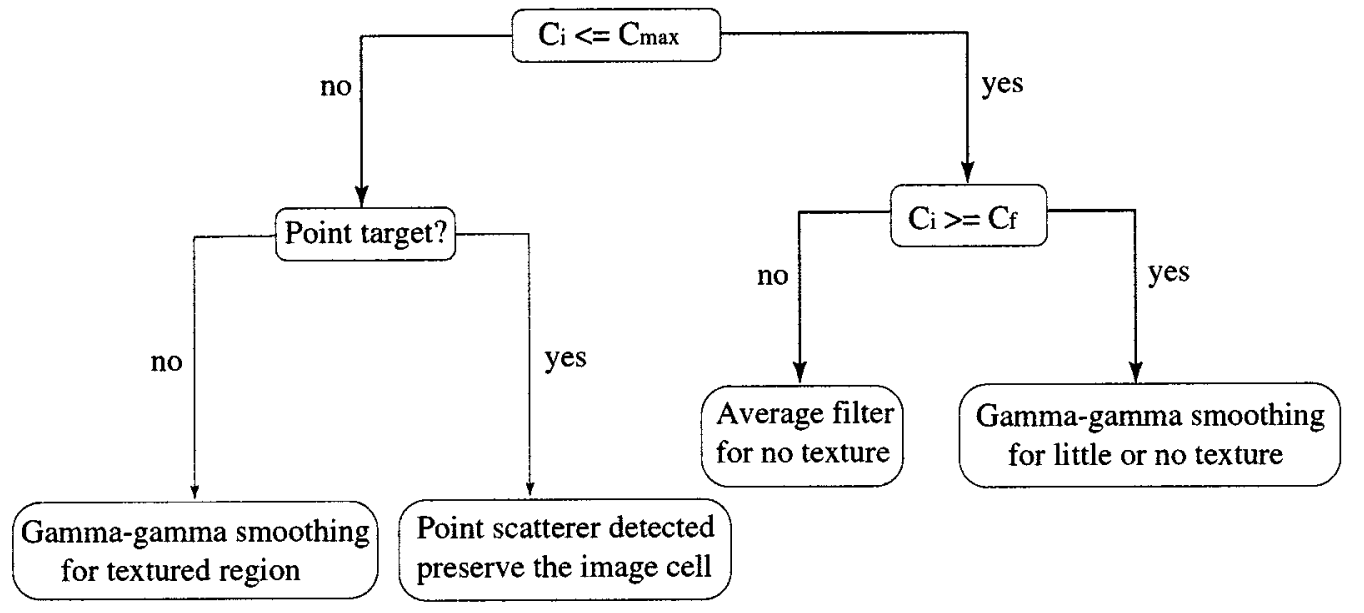

Fig. 2. Gamma-gamma filter with point target detection.

To accommodate this situation, we designed two filters to cover a range of possible speckle conditions. A conservative speckle filter was designed by setting $C_{F}=$ average $\left(C_{I}\right)-$ $2 * \operatorname{std}\left(C_{I}\right)$ and $C_{\max }=\operatorname{average}\left(C_{I}\right)$. An aggressive speckle filter was designed by setting $C_{F}=$ average $\left(C_{I}\right)$ and $C_{\max }=$ average $\left(C_{I}\right)+2 * \operatorname{std}\left(C_{I}\right)$. See Fig. 3 for a pictorial placement of $C_{F}$ and $C_{\max }$ on the $C_{I}$ histogram. Choosing to offset $C_{F}$ and $C_{\max }$ in this manner improved experimental repeatability 


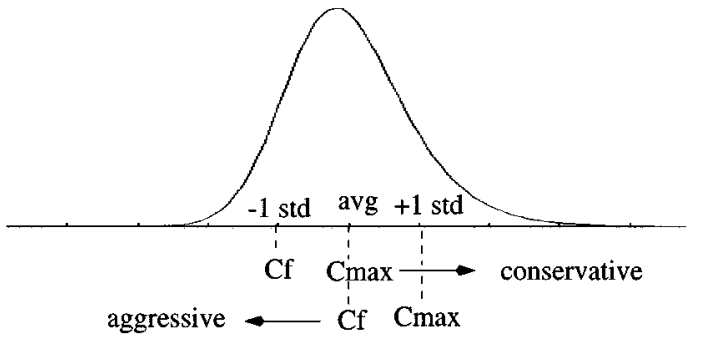

Fig. 3. Conservative and aggressive settings for $C_{F}$ and $C_{\max }$, shown on the $C_{I}$ histogram.

\begin{tabular}{|l|l|l|l|l|l|l|}
\hline & & & $\mathbf{p}$ & & & \\
\hline & & & $\mathbf{p}$ & & & \\
\hline & & $\mathbf{p}$ & $\mathbf{p}$ & $\mathbf{p}$ & & \\
\hline $\mathbf{p}$ & $\mathbf{p}$ & $\mathbf{p}$ & $\mathbf{p}$ & $\mathbf{p}$ & $\mathbf{p}$ & $\mathbf{p}$ \\
\hline & & $\mathbf{p}$ & $\mathbf{p}$ & $\mathbf{p}$ & & \\
\hline & & & $\mathbf{p}$ & & & \\
\hline & & & $\mathbf{p}$ & & & \\
\hline
\end{tabular}

Fig. 4. Those cells marked "p" are cells which may represent a point target, and the other cells are surrounding cells.

when using other datasets. The window size of these filters was $7 \times 7$. A larger window is generally better at reducing speckle, but because the GGMAP filter was also expected to preserve texture, a smaller than usual window size was used.

The threshold for point target detection was set to 0.15 . This threshold is compared to the inverse ratio of the average of those image cells' DN's, which may represent a point target divided by the average of the surrounding image cells' DN's

$$
\frac{\text { average of surrounding image cells }}{\text { average of point target image cells }} \text {. }
$$

See Fig. 4, where "p" represents a cell that may be part of the microwave response to a point scatterer. If the ratio is less than 0.15 , then a point target has been detected. The threshold was chosen by first generating a binary image of a portion of the scene where 1 meant a point target was detected, and 0 meant no point targets were detected. The threshold was varied until most of the point targets, visual to the author, were detected.

\section{B. Grey Level Co-Occurrence Texture Features}

Co-occurrence probabilities, a second-order method for generating texture features, represent the most popular method of characterizing texture in remotely sensed imagery. A brief presentation of the grey level co-occurrence matrix (GLCM) follows, but a more complete explanation is provided by Haralick et al. [4]. The matrix contains the conditional joint probabilities of all pairwise combinations of grey levels given two parameters: interpixel distance $\delta$ and interpixel orientation $\theta$. Following Barber and LeDrew [1], the probability measure can be defined by

$$
\operatorname{Pr}(x)=\left\{C_{i j} \mid(\delta, \theta)\right\}
$$

where $C_{i j}$ (the GLCM) is defined by

$$
C_{i j}=\frac{P_{i j}}{\sum_{i, j=1}^{G} P_{i j}} .
$$

$P_{i j}$ represents the number of occurrences of grey levels $i$ and $j$, and $G$ is the total number of grey levels. The sum in the denominator represents the total number of grey level pairs within a window given a particular $\delta, \theta$. A different GLCM is determined for each $\delta, \theta$. In our work, we assumed that the image texture was isotropic, and we pooled each pair of angles at the same offset into one GLCM, e.g., $0^{\circ}$ and $90^{\circ}$ were pooled into the offset $\delta=1$.

In practice, the image is quantized to a certain number of bits per pixel to save computation time and reduce memory requirements. Here, the quantization level is 256 . Texture statistics are calculated by summing weighted probabilities over the entire GLCM. Given that many of the statistics are correlated [19], only a few statistics are necessary to capture the essential information from the GLCM. In this work, the following three statistics were selected, e.g., Clausi [2]

$$
\begin{aligned}
\text { entropy } & =-\sum_{i, j} P_{i j} \log P_{i j} \\
\text { dissimilarity } & =\sum|i-j| P_{i j} \\
\text { correlation } & =\sum_{i, j} \frac{\left(i-\mu_{x}\right)\left(j-\mu_{y}\right) P_{i j}}{\sigma_{x} \sigma y}
\end{aligned}
$$

where $\left(\sigma_{x}, \sigma y\right)$ and $\left(\mu_{x}, \mu_{y}\right)$ represent the mean and standard deviations in the row and column directions. Here, the co-occurrence probabilities are determined over $15 \times 15$ windows, which represent spatial extents larger than the resolution cells.

\section{Analysis Methods}

The data from the virtual forests were statistically tested with a multivariate analysis of variance (MANOVA) and a Fisher criterion and classified with a weighted Euclidean distance measure.

The MANOVA and Fisher criterion are necessary to form conclusions about the relationship between SAR image texture and the forestry parameters. In a hypothetical situation, where the Fisher criterion failed to indicate strong separability, the MANOVA may still indicate that the texture is significantly different. This would prove that although classification may not be practical, the texture feature vectors are still measuring a change in the texture. In other words, the texture measure is still able to detect a fundamental change in the forest stand properties.

In addition to the MANOVA and the Fisher criterion, a more widely understood measurement, the percentage of windows that were correctly classified with the texture features, was also calculated. Although partially correlated with the Fisher criterion, classification results could support or contradict our hypothesis that SAR image texture contains useful information that a wider audience could understand. Furthermore, the classification scheme makes Gaussian assumptions that the Fisher criterion does not. 
1) MANOVA: MANOVA [5] is able to determine if two datasets have statistically equal or unequal means. For example, if two sets of texture feature data from a virtual forest, identical in every manner but of different height, are proven to have significantly unequal means, then this supports a relationship between texture and the forestry parameter under consideration. In other words, this indicates that a change in height has a corresponding change in texture. The results from a simple classification scheme would not only support the MANOVA results, but also measure the degree of separability between the texture feature classes.

The MANOVA test requires independent samples from two populations. This condition is met by taking samples from independent forest stands (those not adjacent are considered independent). If the sample population size is small, then for a meaningful MANOVA both populations must be multivariate normal and have equal covariance matrices.For our forest stands the sample sizes were adequate (greater than 29, but usually greater than 100) in which case the above assumptions can be relaxed [5]. Nevertheless, a random and infrequent comparison of covariance matrices showed only insignificant differences.

2) Fisher Criterion: The Fisher criterion [5] is a ratio between the interclass distance and the intraclass variation. This measure represents the separability of the texture features. For example, high separability between virtual forest stands with different stocking indicates that texture is a function of stocking. Moreover, the Fisher criterion places no assumptions upon the distribution of the data, unlike the MANOVA test.

3) Classification: A standard weighted Euclidean distance measure was adopted to classify the forest stands. Half the data was used as training data, which produced an estimate of the mean and covariance matrix. The other half was classified. For each unique stand of trees, the multivariate mean and variance were calculated. The decision rule for feature vector $x$, given classes $A$ and $B$, is

$$
\begin{aligned}
x \in & A \operatorname{iff}\left(x-m_{A}\right) S_{A}^{-1}\left(x-m_{A}\right)^{T} \\
& <\left(x-m_{B}\right) S_{B}^{-1}\left(x-m_{B}\right)^{T} \forall A \neq B
\end{aligned}
$$

where $m$ indicates a class mean vector, and $S$ indicates the class covariance matrix. This classification method assumes normality, but because of the large number of samples, this assumption could be relaxed. We should note that we were not attempting to perform an optimal classification, but rather to get a quick estimate to support the MANOVA results. This research seeks to show that forestry parameters are related to texture, so this classification is only a rough procedure that could potentially be improved by testing the normal assumption or choosing another classification scheme without normal assumptions.

\section{Experimental Design}

The following explains how these tests were used. Note that MANOVA test statistics could be replaced with the classification accuracy or Fisher criterion in the following explanation. For a set of virtual forest stands (which compose a virtual forest), MANOVA test statistics for all pairs are calculated and then averaged. For example, take a fictitious
TABLE I

Forest INVENTORY CATEGORIES USED IN THE BRITISH COLUMBIA ForeST

\begin{tabular}{|c|c|c|}
\hline \\
\hline \multicolumn{3}{|c|}{ Species Codes and Classes } \\
\hline 2 & B & Balsam \\
\hline 3 & $\mathrm{C}$ & Western red cedar \\
\hline 4 & $\mathrm{Cy}$ & Yellow cedar \\
\hline 6 & $\mathrm{D}$ & Alder \\
\hline 7 & $\mathrm{~F}$ & Douglas fir \\
\hline 8 & $\mathrm{H}$ & Hemlock \\
\hline 10 & $\mathrm{Pl}$ & Lodgepole pine \\
\hline 11 & $\mathrm{Pw}$ & White pine \\
\hline 12 & $\mathrm{~S}$ & Spruce \\
\hline \multicolumn{3}{|c|}{ Age Codes and Classes } \\
\hline & \multicolumn{2}{|c|}{$1-20$ years } \\
\hline 2 & \multicolumn{2}{|c|}{$21-40$ years } \\
\hline 3 & \multicolumn{2}{|c|}{$41-60$ years } \\
\hline 4 & \multicolumn{2}{|c|}{$61-80$ years } \\
\hline 5 & \multicolumn{2}{|c|}{$81-100$ years } \\
\hline 6 & \multicolumn{2}{|c|}{$101-120$ years } \\
\hline 7 & \multicolumn{2}{|c|}{$121-140$ years } \\
\hline 8 & \multicolumn{2}{|c|}{$141-250$ years } \\
\hline g & \multicolumn{2}{|c|}{$250+$ years } \\
\hline \multicolumn{3}{|c|}{ Height Codes and Classes } \\
\hline 1 & \multicolumn{2}{|c|}{$1-10 \mathrm{~m}$} \\
\hline 2 & \multicolumn{2}{|c|}{$10.1-20 \mathrm{~m}$} \\
\hline 3 & \multicolumn{2}{|c|}{$20.1-30 \mathrm{~m}$} \\
\hline 4 & \multicolumn{2}{|c|}{$30.1-40 \mathrm{~m}$} \\
\hline 5 & \multicolumn{2}{|c|}{$40.1-50 \mathrm{~m}$} \\
\hline 6 & \multicolumn{2}{|c|}{$50.1-60 \mathrm{~m}$} \\
\hline 7 & \multicolumn{2}{|c|}{$60.1-70 \mathrm{~m}$} \\
\hline 8 & \multicolumn{2}{|c|}{$70.1-80 \mathrm{~m}$} \\
\hline \multicolumn{3}{|c|}{ Stocking Codes and Classes } \\
\hline \multicolumn{3}{|c|}{1 dense } \\
\hline \multicolumn{3}{|c|}{2 normal } \\
\hline \multicolumn{3}{|c|}{3 open } \\
\hline
\end{tabular}
INDUSTRY FOR AGE, HEIGHT, AND STOCKING

set of virtual forest stands $\{\mathrm{H}-2-3-3, \mathrm{H}-3-3-3, \mathrm{CB}-2-3-3$, CB-3-3-3, C-2-3-4\} where the first letter(s) represent the species (or species composition in the case of two letters), the first number represents age class, the second number represents height class, and the last number represents stocking class (species-age-height-stocking). The codes are provided in Table I. The first virtual forest stand in the set has species code of $\mathrm{H}$, age class 2 , height class 3 , and stocking class 3 . For the species virtual forest, MANOVA test statistics would be calculated from only two comparisons: $\mathrm{H}-2-3-3$ with $\mathrm{CB}-2-3-3$ and $\mathrm{H}-3-3-3$ with $\mathrm{CB}-3-3-3$. In these comparisons, only the species changes are used, not the age, height, or stocking. The two test statistics would be averaged and plotted above the species $x$-label on the graphs in the results section. Stand C-2-3-4 could not be used with $\mathrm{H}-2-3-3$ or CB-2-3-3 because the height is different. For another example, consider the age virtual forest, where two test statistics would be calculated by using $\mathrm{H}-2-3-3$ with $\mathrm{H}-3-3-3$ and $\mathrm{CB}-2-3-3$ with $\mathrm{CB}-3-3-3$ in the average. Nothing changes but the age.

Within the $15 \times 15$ pixel window we estimated, the three GLCM features at six different offsets $[(1,0),(1,1),(2,0),(2,2)$, $(3,0),(3,3)]$. These correspond to linear scales in meters: 3.125 , 
Raw Image

MANOVA vs Distance for Species
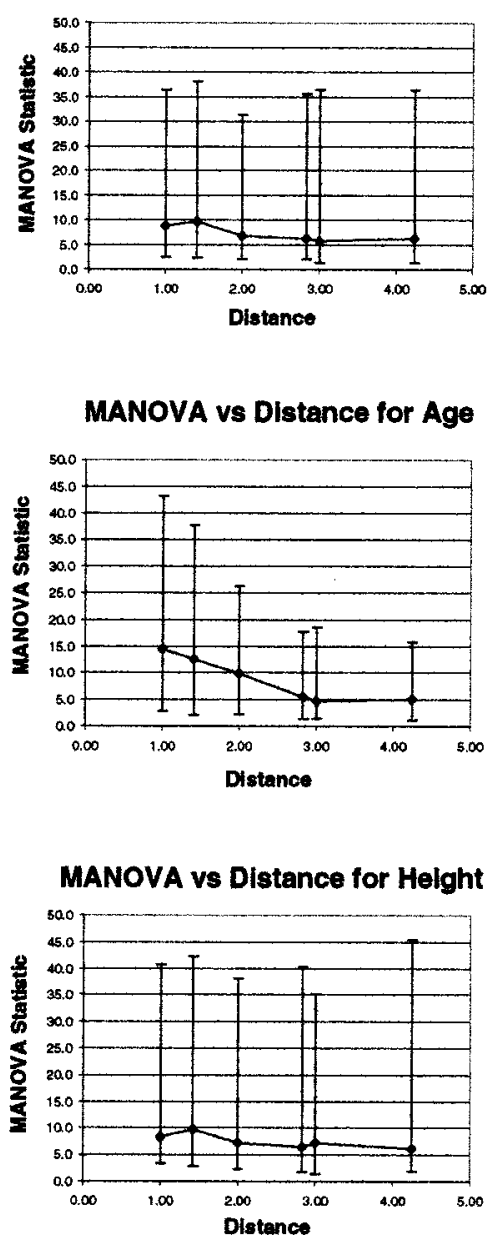

MANOVA vs Distance for Stocking

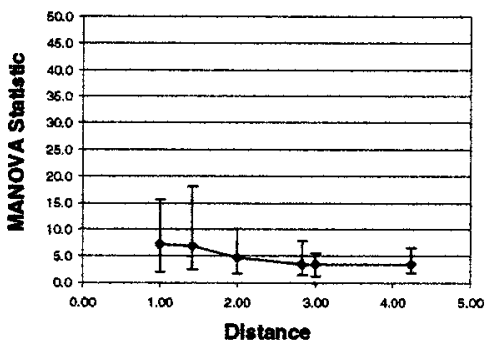

Conservatively Filtered Image

MANOVA vs Distance for Specles

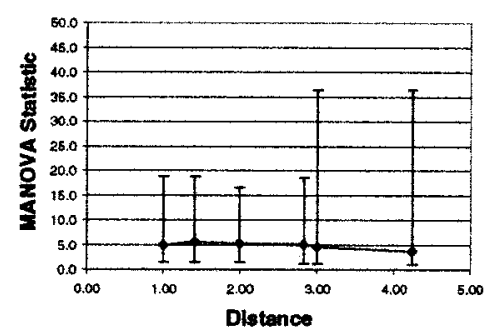

MANOVA vs Distance for Age

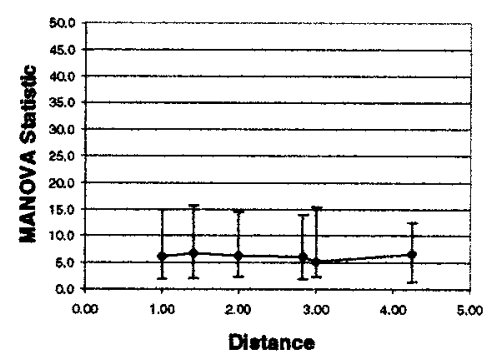

MANOVA vs Distance for Helght

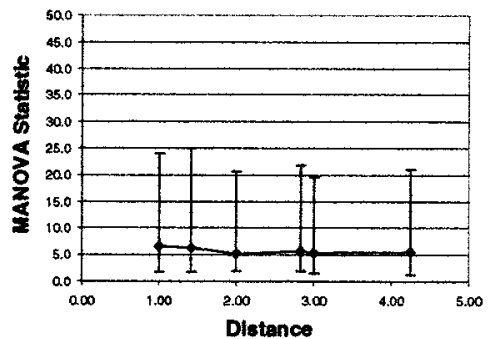

MANOVA vs Distance for Stocking

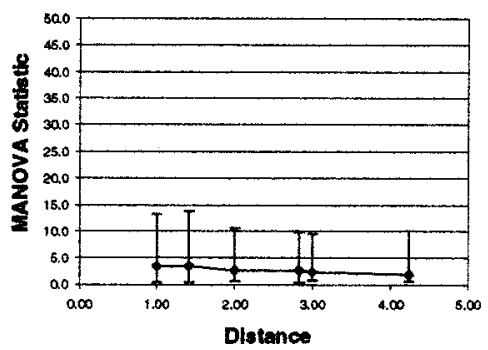

Aggressively Filtered Image

MANOVA vs Distance for Species

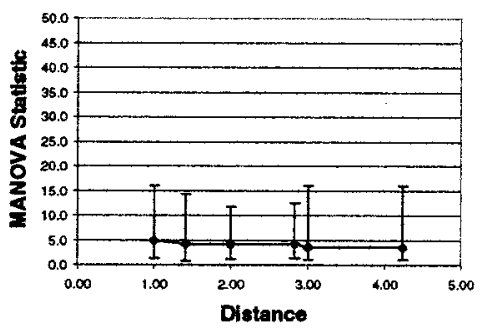

MANOVA vs Distance for Age

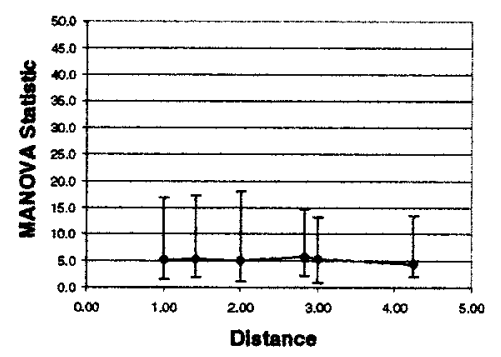

MANOVA vs Distance for Helght

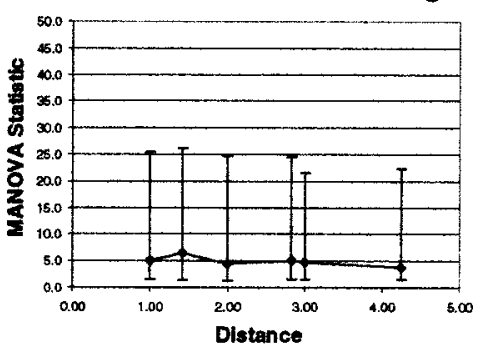

MANOVA vs Distance for Stocking

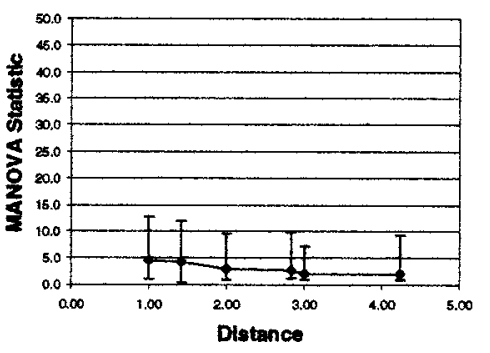

Fig. 5. Results of the MANOVA test. Each column represents a different speckle filtering case, and each row represents a different forest parameter.

$4.419,6.250,8.839,9.375$, and $13.258 \mathrm{~m}$. We expect to find the information contained in the texture, as estimated through the three statistical parameters noted above, to decline as the offset increases.

\section{RESULTS}

Our results are shown in three graphs: MANOVA results are in Fig. 5, Fisher distance results are in Fig. 6, and the Bayesian classification results are in Fig. 7. These graphs have a black dot at the mean value of the statistic and an error bar. The error bars represent the 16 and 84 percentiles. This means that $16 \%$ of the samples are below the bottom error bar, $16 \%$ of the samples are above the top error bar, and $68 \%$ of the data is contained within the error bars. The error bars should give a useful impression of the uncertainty.

\section{A. MANOVA}

A larger MANOVA test statistic indicates a stronger relationship between the image texture and the forest parameter. If there is more information in image texture near the scale of the 


\section{Raw Image}

Fisher vs Distance for Species

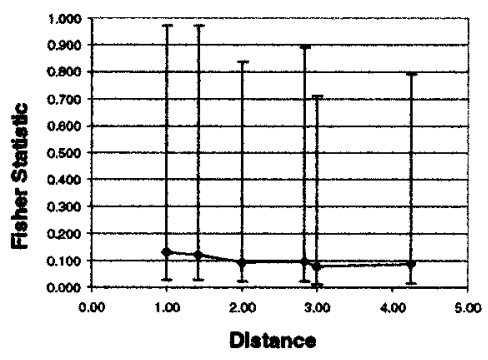

Fisher vs Distance for Age

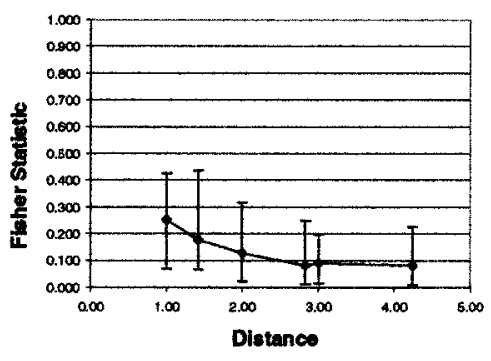

Flsher vs Distance for Helght

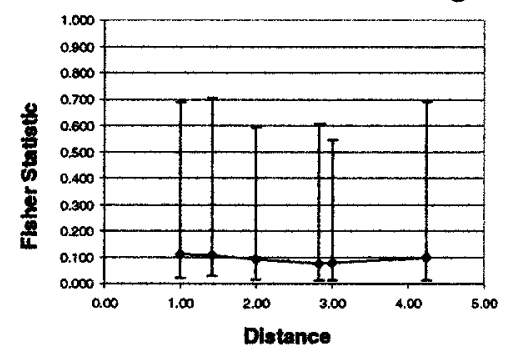

Fisher vs Distance for Stocking

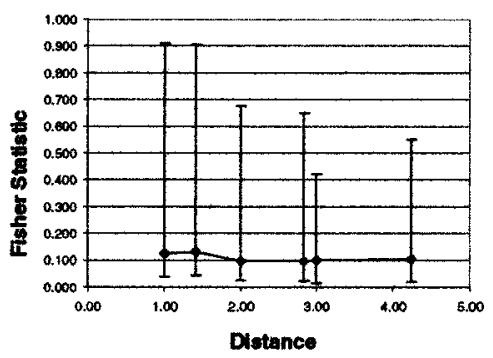

Conservattvely Filtered Image

Fisher vs Distance for Specles

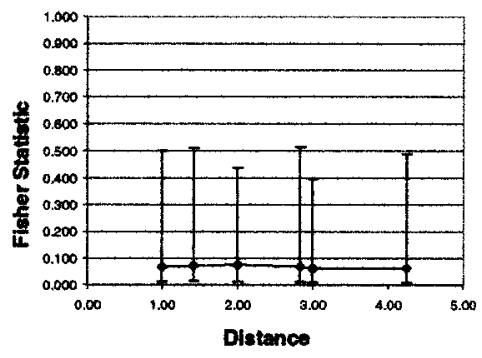

Fisher vs Distance for Age

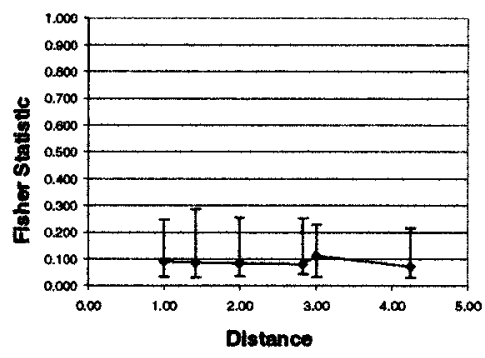

Fisher vs Distance for Height

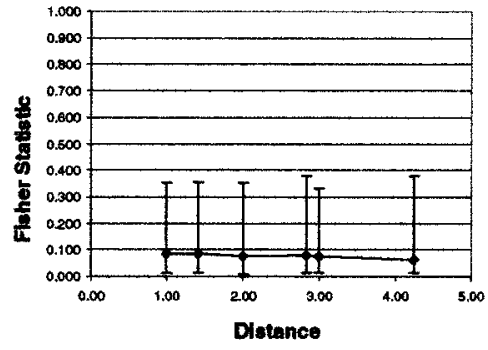

Fisher vs Distance for Stocking

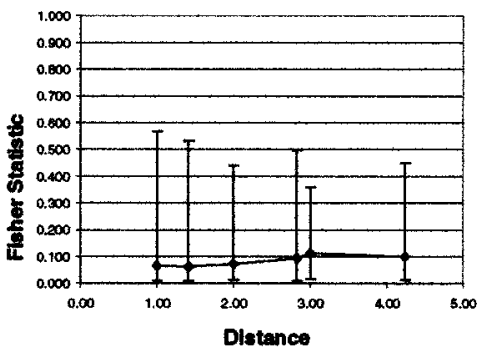

Aggressively Filtered Image

Fisher vs Distance for Species

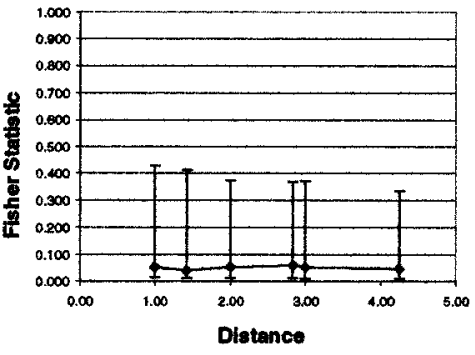

Fisher vs Distance for Age

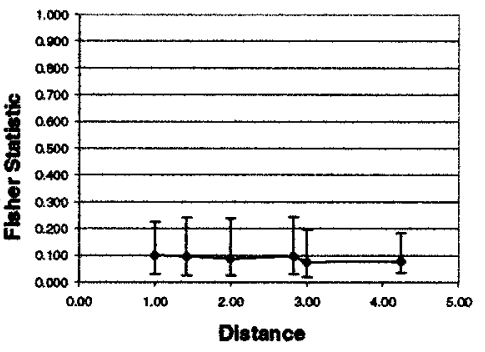

Flsher vs Distance for Helght

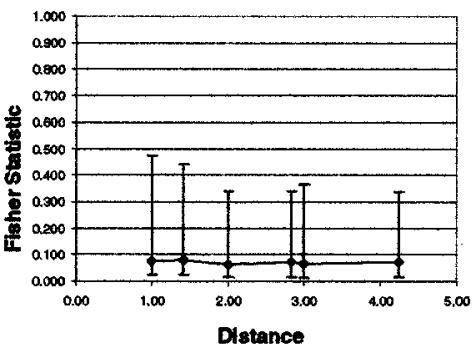

Fisher vs Distance for Stocking

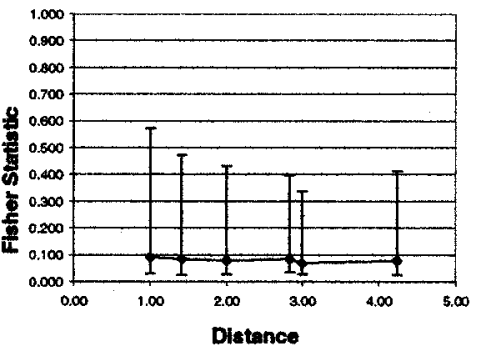

Fig. 6. Results of the Fisher Distance Test. Each column represents a different speckle filtering case, and each row represents a different forest parameter.

resolution cell than at larger scales, then we would expect the MANOVA test statistic to decline with distance. Our hypothesis is that the speckle filtering is destroying backscatter texture. Hence, if we see a decline in the MANOVA test statistic with distance for the raw image, we should see a relatively flat response for the filtered images.

Our hypothesis appears to be supported for all forest parameters shown in Fig. 5. The strongest support is for age, where the statistic decreases by $60 \%$ between 1 and 3 pixels, after which, it remains flat. For the filtered images, the statistic remains essentially flat with distance. This applies to a lesser extent for the other forest parameters. However, in each case, the MANOVA statistic for the raw image decreases with distance. The filtered response remains flat for height, and decreases slightly for species and stocking, indicating that a small amount of backscatter texture is being preserved by the filter. 
Raw Image

Bayes vs Dlstance for Species

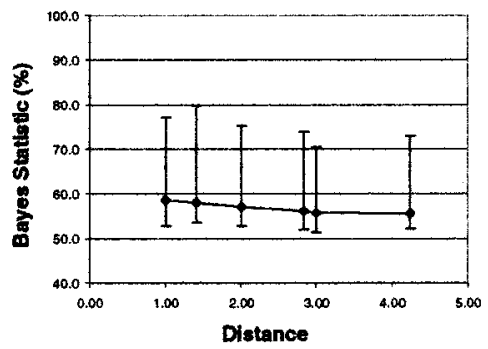

Bayes vs Distance for Age

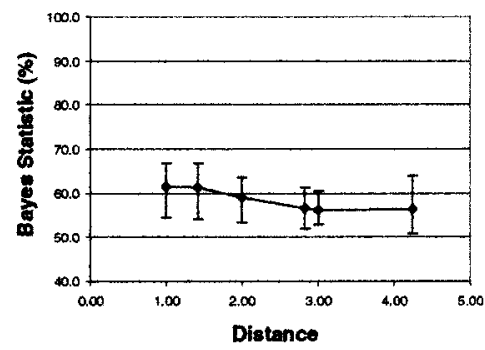

Bayes vs Distance for Helght

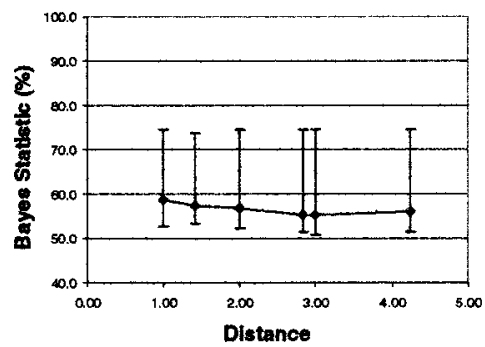

Bayes vs Distance for Stocking

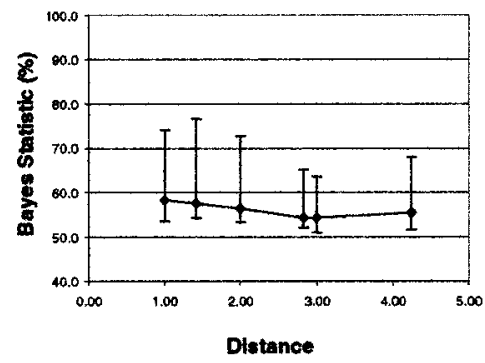

Conservatively Filtered Image

Bayes vs Dlstance for Specles

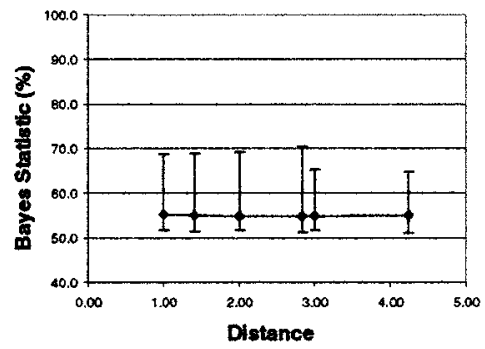

Bayes v8 Dlstance for Age

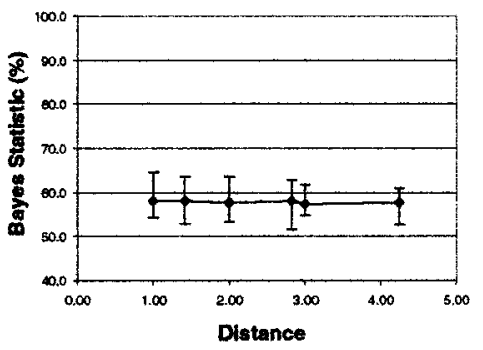

Bayes vs Dlstance for Height

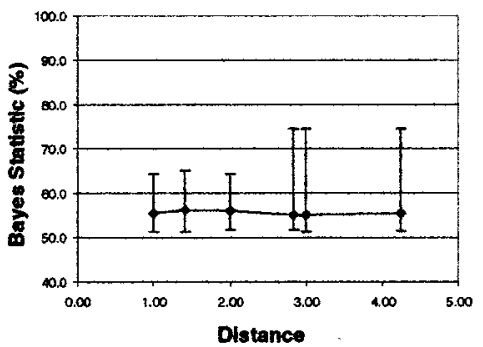

Bayes vs Distance for Stocking

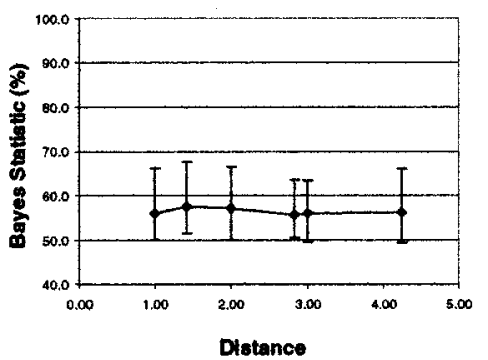

Aggressively Filtered Image

Bayes vs Distance for Specles

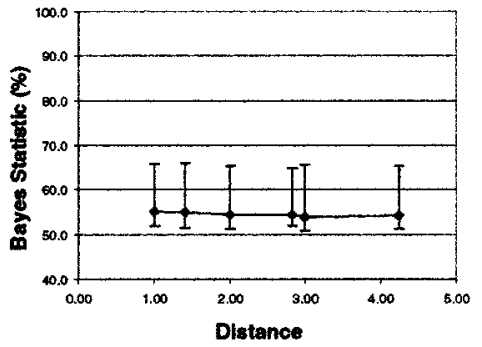

Bayes vs Distance for Age

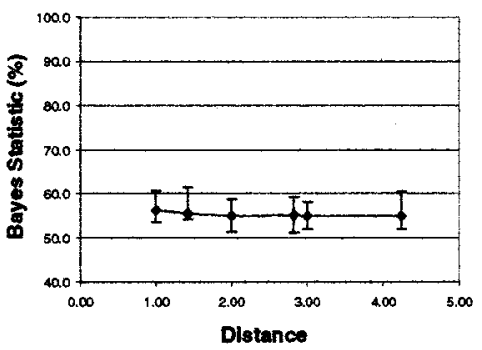

Bayes vs Distance for Helght

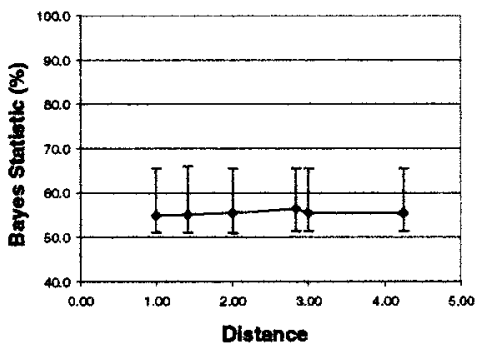

Bayes vs Distance for Stocking

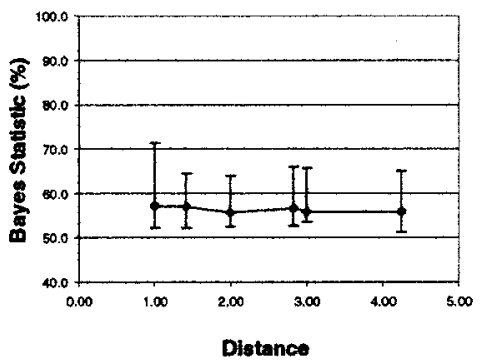

Fig. 7. Results of the Bayesian Classification. Each column represents a different speckle filtering case, and each row represents a different forest parameter.

Interestingly, there is very little difference between the conservative filter and aggressive filter.

\section{B. Fisher Distance}

For the Fisher distance, a higher statistic indicates greater separability between the categories of the forest parameter based on image texture. Hence, if there is more information in resolution-scale texture, then we would again expect the Fisher statistic to decline with distance. If this occurs in the raw image, then our hypothesis is supported if the filtered images yield a flat response.

Fig. 6 suggests that our hypothesis is supported strongly for age. The statistic declines between 1 and 3 pixels for the raw image and remains flat against distance for the filtered images, indicating that texture-based separability was enhanced at short distances and that this information was not available in the filtered images. There is a very slight decrease in the statistic for 
species and stocking, while the filtered responses for these parameters remain flat or increase slightly. The height result is essentially flat for all images, indicating no scale dependence for this parameter. We are inclined to suggest that the scale dependence for species and stocking is negligible.

\section{Bayes Classifier}

The results of the simple Bayes classifier are shown in Fig. 7. The points on these graphs indicate the percentage of pixels for the species, age, height, and stocking parameters that were correctly predicted using image texture. Again, we observe the strongest scale dependence for the age parameter and that the filtered images do not retain this resolution-scale texture information. The three other parameters all decline with distance, indicating some degree of scale-dependent texture information, and the filtered responses are all either flat, indicating no scale dependence, or slightly increasing, indicating that the texture has been suppressed at smaller scales.

\section{Uncertainty}

It is clear that the uncertainty of our results, as indicated by the error bars, may weaken our ability to make assertions in this research. In the MANOVA and Fisher distance results, the error bars for the unfiltered data are, in some cases, much larger than for the filtered results. This is an issue that needs to be investigated further to understand whether the uncertainty exists for all categories of each parameter or whether certain categories contribute more uncertainty than others. However, given the non-Gaussian distribution of the test statistics, the mean value is very conservative, and assertions based on this mean value will thus be conservative.

\section{CONCLUSIONS}

We have found evidence that supports the contention that the backscatter texture bearing information on forest stand characteristics in Radarsat fine mode image data is scale dependent. We have observed a decrease in the ability the image texture to predict forest parameters as the texture scale increases. This suggests that there is considerable information in the texture at scales near that of the system resolution. Our results also indicate that the adaptive speckle filtering algorithm developed by Lopes et al. [12] is not preserving this small scale texture. This is certainly consistent with the inherent assumptions of the product data model upon which their algorithm is based. It suggests that the product model might not be appropriate for these data and this application. However, further experiments would be necessary to confirm this hypothesis.

\section{REFERENCES}

[1] D. Barber and E. LeDrew, "SAR sea ice discrimination using texture statistics: A multivariate approach," Photogramm. Eng. Remote Sensing, vol. 57, pp. 385-395, 1991.

[2] D. Clausi, "Texture segmentation of sar sea ice imagery," Ph.D. dissertation, Univ. Waterloo, Dept. Syst. Design Eng., Waterloo, Ont., Canada 1996.
[3] V. S. Frost, J. A. Stiles, K. S. Shanmugan, and J. C. Holtzman, "A model for radar images and its application to adaptive digital filtering of multiplicative noise," IEEE Trans. Pattern Anal. Machine Intell., vol. 4, pp. 157-165, Feb. 1982.

[4] R. M. Haralick, K. Shanmugan, and I. Dinstein, "Textural features for image classification," IEEE Trans. Syst., Man, Cybern., vol. 3, pp. 610-621, Dec. 1973.

[5] R. A. Johnson and D. W. Wichern, Applied Multivariate Statistical Analysis, 3rd ed. Englewood Cliffs, NJ: Prentice-Hall Inc., 1992.

[6] D. T. Kuan, A. A. Sawchuk, T. C. Strand, and P. Chavel, "Adaptive noise filter for images with signal-dependent noise," IEEE Trans. Pattern Anal. Machine Intell., vol. 7, pp. 165-177, Feb. 1985.

[7] — "Adaptive restoration of images with speckle," IEEE Trans. Acoust., Speech, Signal Processing, vol. ASSP-35, pp. 373-383, Mar. 1987.

[8] H. J. H. Kux and C. M. Henebry, "Multi-scale texture in SAR imagery: Landscape dynamics of the Pantanal," in IGARSS, Pantanal, Brazil, 1994, pp. 1069-1071.

[9] J.-S. Lee, "Speckle analysis and smoothing of synthetic aperture radar images," Comput. Graph. Image Process., vol. 17, pp. 24-32, 1981.

[10] A. Lopes, E. Nezry, R. Touzi, and L. Laur, "Maximum a posteriori speckle filtering and first order texture models in SAR images," in Int. Geosci. Remote Sensing Symp.. Vancouver, B.C., Canada, 1990.

[11] _ , "Structure detection and statistical adaptive speckle filtering in SAR images," Int. J. Remote Sensing, vol. 14, no. 9, pp. 1735-1758, 1993.

[12] A. Lopes, R. Touzi, and E. Nezry, "Adaptive speckle filters and scene heterogeneity," Trans. Geosci. Remote Sensing, vol. 28, pp. 992-1000, Nov. 1990.

[13] A. Luckman, G. Groom, and J. Baker, "Forest age discrimination from texture measures of SAR imagery," in Igarss 1994, 1994, pp. 104-107.

[14] P. Murtha, "Airborne SAR studies of north Vancouver Island rainforests," Can. J. Remote Sensing, vol. 22, no. 2, pp. 175-183, 1996.

[15] _ _ "Radar imaging of natural systems (RAINS)," in Ger '97, 1997.

[16] C. Oliver and S. Quegan, Understanding Synthetic Aperture Radar Images. Boston, MA: Artech House, 1998.

[17] T. Pultz and R. Brown, "Image classification of agricultural targets using first and second-order statistics," Can. J. Remote Sensing, vol. 13, no. 2, pp. 85-91, 1987.

[18] E. Rignot and R. Kwok, "Characterization of spatial statistics of distributed targets in sar data," Int. J. Remote Sensing, vol. 14, no. 2, pp. 345-363, 1993.

[19] F. Ulaby, F. Kouyate, B. Brisco, and T. H. L. Williams, "Textural information in SAR images," Trans. Geosci. Remote Sensing, vol. GE-24, pp. 235-245, Mar. 1986.

[20] J. Wiebe, M. J. Collins, D. Clausi, and P. Murtha, "Relating sar image texture to forestry inventory parameters,", to be published.

Michael J. Collins (S'87-M'93) received the B.Sc.Eng. degree in survey engineering from the University of New Brunswick, Fredericton, N.B., Canada, in 1981, the M.Sc. degree in physical oceanography from the University of British Columbia, Vancouver, B.C., Canada, in 1987, and the Ph.D. degree in earth and space science from York University, North York, Ont., Canada, in 1993. His $\mathrm{Ph} . \mathrm{D}$. research was a theoretical and experimental analysis of the response of a synthetic aperture radar to sea ice.

He is currently an Associate Professor in the Department of Geomatics Engineering, University of Calgary, Calgary, Alta, Canada. Prior to coming to Calgary, he was a Faculty Member with the University of Maine, Farmington, and the University of New Brunswick. He was a Project Scientist with the Institute for Space and Terrestrial Science, Toronto, Ont., and an Engineer with the McElhanney Group, Vancouver and Calgary. He has also served as a Consultant to government and industry on various aspects of remote sensing. His research interests are in several aspects of microwave remote sensing, including coherent imaging of distributed scenes and the fusion of active and passive microwave measurements, multisensor imaging of polar and vegetated regions, and the geometric properties of remote sensing systems.

Dr. Collins is a Member of the Remote Sensing Society (U.K.), the American Geophysical Union, and Sigma Xi. 


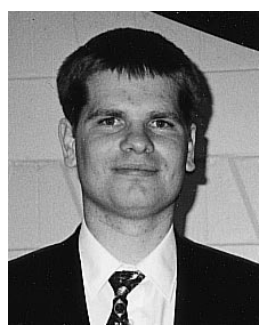

Jonathan Wiebe received the B.A. degree in applied mathematics from the University of Waterloo, Waterloo, Ont., Canada, in 1995, and the M.S. degree in geomatics engineering from the University of Calgary, Calgary, Canada, in 1998.

He is currently a GPS (Global Positioning System) Engineer with NovAtel Inc., Calgary, Alta. His remote sensing research interests include synthetic aperture radar texture analysis and speckle filtering.

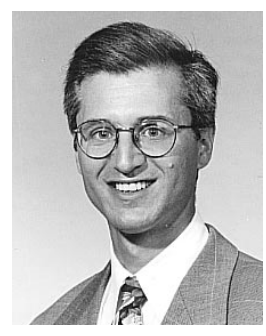

David A. Clausi received the B.A.Sc., M.A.Sc., and $\mathrm{Ph} . \mathrm{D}$. degrees in engineering from the University of Waterloo, Waterloo, Ont., Canada, in 1990, 1992, and 1996, respectively.

He was with Mitra Imaging, Inc., and was then an Assistant Professor in the Department of Geomatics Engineering, University of Calgary, Calgary, Alta., Canada. Since 1999, he has been with the University of Waterloo, Ont., Canada, where he is an Assistant Professor in Systems Design Engineering. His research interests include automated interpretation, digital image processing, texture analysis, and pattern recognition with applications in remote sensing and medical imaging. 